\title{
Analysis of Interior Space Design and Visual Artistic Effect
}

\author{
Wang Qian \\ Academy of Arts, Xi'an Fanyi University, Shaanxi, China, 710105 \\ Keywords: Interior Space; Psychological Need; Visual Art; Relationship \\ Abstract: Interior space is the basic living environment structure of human beings, and it is \\ the material environment for people to live and work. Therefore, the design of interior \\ space involves the relationship between people and space, and often reflects the behavioral \\ and psychological needs of people from the side. With the improvement of material living \\ standards, people's demand for interior space design has long been no longer limited to a \\ simple place, but a higher level of design connotation and concept, more in line with \\ personal life needs. In the design process, designers in this field have to consider the visual \\ artistic effect in order to make the interior space perfect. Therefore, designers should not \\ only consider from the applicability and practicability of space, but also need to consider \\ from the aspects of color matching, plane composition and visual perception. Besides, it is \\ also necessary to use the formal language of art to combine interior design with visual \\ artistic effect to design works that better meet the psychological needs of contemporary \\ people. Through the exploration and research of interior space design and visual artistic \\ effect, this paper discusses the meaning and design principles of interior space design and \\ visual artistic effect on interior space design. In addition, this paper also discussed the \\ embodiment of visual artistic effect in interior space design through the actual example \\ analysis.
}

\section{The Meaning and Design Principle of Interior Space Design}

The interior space design refers to the use of material means by the designer to create an indoor environment that combines the functional and aesthetic characteristics of the building and meets the material and spiritual needs of people according to the environment in which the building is located and other corresponding standards. At the same time, it can also reflect the characteristics of the building itself and the human atmosphere.

As an extension of architectural design, interior space design is the main way to improve building functions on the basis of architecture. Its design principles are mainly reflected in four points. The first principle is functionality and applicability. When transforming indoor space, rational use of space and meeting the practical use and specific needs of space is the primary goal of interior space design, which is to make people and space be coordinated and satisfied to the greatest extent. The second principle is economic rationality. As the name implies, that is, while innovating and improving the taste of the interior, we must fully consider the principles of economic application, be practical, and not spend extra money on materials and energy. The third is the 
principle of coordination with the objective environment. Although interior space design is an extension of architectural design, both are subject to environmental and other objective factors. Therefore, in the design, we must emphasize the principle of coordination with the objective environment, so as to do a good job in the use of materials and space. The fourth principle is the overall design thinking. As the component of architectural design, interior space design should take into account the overall design thinking, coordinate with the overall characteristics of the building in terms of spatial form and architectural style, and build a more harmonious living or working environment.

At present, the development of interior space design field has been continuously transformed with the economic growth of our country. Whether it is the design concept, the design plan, or the layout and utilization of the space has been further improved. The diversified interior space design style and diversified aesthetic understanding have contributed to the in-depth development of interior space design itself. In the process of interior design, how to make good use of space and create a good visual artistic effect is the primary development goal in the field.

\section{The Function of Visual Artistic Effect to Interior Space Design}

Visual perception is one of the most basic perceptions of people to things, and the most important way to convey the communication between people, people and the environment. Today, visual art is widely used in various industries, which not only greatly promotes the development of innovation in various fields, but also activates its own diversified development.

The field of visual art in the traditional definition does not explicitly point out its relationship with the interior space design, but today, interior space design is full of visual art. It can not only be reflected from the angles intuitive images, but also enhance the atmosphere and artistic conception of indoor space through visual experience, thus becoming an important way to convey emotion and aesthetic perception. For example, when visual art uses a wall as a carrier, the wall will be like a canvas as used in two-dimensional painting, and its creative means will be more direct. Moreover, the wall surface has a large plasticity and can be expressed by various art forms. Different wall surfaces are affected by different materials, shapes, colors, etc., and can be shaped into a visual experience with a large space for exertion. In addition, when visual art uses the ground as a carrier, the designer can achieve the purpose of creating an indoor space atmosphere by the color of the overall space, the matching of the materials, and the arrangement of the composition. For example, the color of the floor can reflect the color of the corresponding place. The composition of the point, line and surface in the floor can create the atmosphere and temperament of the whole environment. The material of the floor can reflect the cultural background of the indoor space. Moreover, when visual art is based on the ceiling, the designer needs to consider the importance of the ceiling in the interior design during the design process. The ceiling is the most direct and important part of improving the overall spiritual quality of the building and shaping the interior space. Due to the great flexibility of the ceiling design, many designers will make bold ideas in space design, which requires full consideration of the visual art of modeling and composition in architecture and space. For example, the use of lights in the design of the ceiling enhances the sense of form and composition of the overall design.

In summary, visual artistic effect is indispensable in the design of interior space. It contributes to the creation of an environmental atmosphere and can greatly enhance people's aesthetic feelings about space. Moreover, people's psychological and emotional factors can be expressed in an abstract way, thus fully satisfying people's psychological needs for the environment. 


\section{The Combination of Interior Space Design and Visual Art}

The combination of interior space design and visual art is reflected in many aspects. The following two examples of the combination of interior space design and visual artistic effect will be discussed from two more common perspectives of styling and color.

\subsection{Discuss from the Perspective of Styling}

Design activities are not specific operation to a specific phenomenon, but have a certain comprehensive nature, and their generation often begins with a shape. Everything has the existence of a figurative form, which is visible and touchable. When a person consciously creates an image, the shape is created. By arranging space for the design of the space, the designer consciously breaks its inherent form and can visually give a strong sense of design.

To emphasize innovation and function in interior space design, we must first give full play to the characteristics of the design. We can use figurative, abstract, symbolic and other means to create simple, clear, retro, mysterious and other artistic styles, in order to enhance the tension and personality of the space.

However, there are also some architectural designs that overly consider the sense of architecture and neglect the internal rational structure. The design gradually moves from the outside to the inside in a passive direction, which leads to the use of unreasonable problems in the space, wasting materials or space. Therefore, when designers use the sense of style of visual art to design interior space, it is necessary to consider the combination of the sense of style with the rationality of space. For example, the renovation of some abandoned factories, theaters or large public places, these places have special shapes related to the times or culture. If we can carry out reasonable space processing on this basis, we can meet the use function and aesthetic needs of indoor space to a large extent. For example, the old factory buildings in many cities have been transformed into art districts, which not only enable the spacious and large spaces to be reasonably distributed into various art studios, but also bring more rich characteristics of the times and artistic features to the art district.

\subsection{Discuss from the Perspective of Color}

Color is the most important part of interior space design. It not only plays a key role in people's aesthetic consciousness, but also can convey the perception of human beings through the regulation and performance of the atmosphere, and at the same time it will have corresponding psychological and physiological effect on people. Therefore, if the color can be used reasonably in the interior space design, it can not only make up for the inherent defects and shortcomings of the space structure, but also determine the emotional feedback and behavioral response that the space can bring. For example, the physical effect of color can adjust the space at the right place, changing the visual sense of volume and area in the interior space. Another example is that the warmth and shade of the hue are also the main channels for expressing the personality of the space. Generally speaking, warm colors give people the impression of liveliness and warmth, while cool colors give people a smooth inward, soft and cold impression. Dark colors give people a subtle and reserved, quiet and deep impression, while light colors give people a impression of clear, straightforward and sunny. On the other hand, colors can also play a role in psychological perception of temperature. For example, in warm areas, cool colors such as blue, purple, and green are used, while in cold areas, warm colors such as red, warm, and orange are used. However, the temperature is affected by the seasonal changes, and at this point, the designer needs to adapt to the local conditions and choose the appropriate color scheme.

In addition, the psychological hint of colors also plays an important role in the design of interior 
space. For example, orange has a psychological suggestion to promote appetite, and blue has psychological hints that make people think. Besides, high purity and saturation of colors are too easy to give people a hint of inflammatory, but too singular and low purity color is easy to bring people with a boring and empty psychological hints. Therefore, the designer should carefully study the psychological suggestion of colors when choosing color matching. Besides, designers should consider according to the occupant's identity, age, personality, social experience and other comprehensive factors to achieve the satisfaction of spiritual needs through visual effect.

\section{The Development Trend of the Combination of Interior Space Design and Visual Art}

In recent years, with the continuous improvement of people's living standards and the enhancement of aesthetic ability, interior space design and appreciation of visual art have diversified development trends. Therefore, in the actual design, we can't completely separate the two, but we must try our best to achieve unity and harmony and complement each other. The Chinese style of interior space design is currently a highlight. Many public buildings and space are constantly being updated in the design of Chinese style. The visual arts expression elements of the Chinese style are also constantly enriched, from traditional Chinese knots to more abstract Chinese cultural totems are used in the combination and innovation of space design. In the development trend of interior space design and visual are integration, it is not difficult to study that the proportion of cultural factors is on the rise. Therefore, the entry point and innovation of interior space design and visual art still need to find inspiration in traditional culture and cultural innovation. A good design or a visual impact is a burst of cultural heritage seeing from the source. The combination with culture is the inevitable trend and the only way to combine interior space design and visual art, and it is also the true return of art.

\section{Conclusion}

In general, interior space design is full of visual art. The use and performance of visual artistic effect is one of the important channels for interior design to create and perfect space. However, each design needs to combine visual art with the actual situation of space to truly bring out the advantages of interior design.

\section{References}

[1] Zhang Shuhong. Introduction to Interior Design [M]. Wuhan: Huazhong University of Science and Technology Press, 2017.

[2] Yin Zhixian. Fashion Home [J]. Beijing: Fashion Home Magazine, 2004.

[3] John Berger (English). Visual Art Appreciation [M]. Business Press, 1994.

[4] Wen Jian. Interior Color, Furniture and Furnishings Design [M]. Tsinghua University Press, 2007.

[5] Liu Ke. Study on Landscape Art Design of Urban Historical and Cultural Blocks [D]. Southwest Jiaotong University, 2016.

[6] Tian Yao. Study on the Hakka Culture of Gannan in the Perspective of Art Design [D]. Gannan Normal University, 2015.

[7] Yao Weiwei. Research on Visual Design Comfort Based on OCULUSVR Panoramic Stereo Video [D]. Beijing Jiaotong University, 2016.

[8] Zhang Jike. Research on the Future Development Direction of Art Design Education in Colleges and Universities [D]. Dalian Polytechnic University, 2015.

[9] Dong Xuan. The Application of Abstract Art Language in Modern Graphic Design [D]. Hebei Normal University, 2016.

[10] Chen Sijie. Application Practice and Research of Ecological Concept in Interior Design [J]. Popular Literature, 2017 (22): 95-96.

[11] Wu Si. Research on the Construction of the Resource Library of the Design Drawing of Interior Design Specialty 
[J]. Popular Literature, 2017 (22): 225-226.

[12] Wu Huijuan. Application Research of Ecological Concept in Interior Design [J]. Journal of Suzhou Education Institute, 2017, 20 (06): 167-168. 